\title{
Luminosity Measurements in ATLAS and CMS during pp data taking at LHC
}

\author{
Sara Valentinetti ${ }^{* \dagger}$ \\ University of Bologna and INFN \\ E-mail: sara.valentinetti@cern.ch
}

\begin{abstract}
A precise measurement of luminosity is a key component of both the ATLAS and CMS physics programmes at LHC: its uncertainty is one of the dominant systematics for both Standard Model cross section measurements and enters new physics searches. The different strategies used by the two collaborations to assess the luminosity and the methods to absolutely calibrate it are here described, together with the dominant systematic uncertainties. The preliminary luminosity uncertainty obtained for 2017 data is $2.4 \%$ for ATLAS and $2.3 \%$ for CMS.
\end{abstract}

Sixth Annual Conference on Large Hadron Collider Physics (LHCP2018)

4-9 June 2018

Bologna, Italy

\footnotetext{
*Speaker.

${ }^{\dagger}$ On behalf of the ATLAS and CMS Collaborations.
} 


\section{Introduction}

Precision measurement of the luminosity delivered to the experiments as ATLAS [1] and CMS [2] by the LHC is important for a variety of reasons. Online, the luminosity measurement provides realtime feed-back on the LHC performance and operation, as well as to experiment operations for tasks such as measurements and adjustement of trigger prescale factors. Offline, the uncertainty on the luminosity is a systematic for all Standard Model cross-section measurements and for new discoveries; for some precise measurements it can be dominant. During the whole LHC running period many steps forward have been made in the comprehension of the uncertainties related to luminosity monitoring and calibration, which led to an unprecedented accuracy at hadron colliders. In the following, after a short overview on luminosity measurement methodology, the principal monitors of both ATLAS and CMS are described, together with the method adopted to absolutly calibrate the luminosity and the main systematics affecting that measurement.

\section{Luminosity Overview}

The luminosity of a pp collider can be expressed as

$$
L=\frac{R_{\text {inel }}}{\sigma_{\text {inel }}}
$$

where $R_{\text {inel }}$ is the rate of inelastic collisions and $\sigma_{\text {inel }}$ is the pp inelastic cross section. If a collider operates at a revolution frequency $f_{r}$ and $n_{b}$ bunches cross at the interaction point, each of which has an identification number called BCID, this expression can be rewritten as

$$
L=\frac{\mu n_{b} f_{r}}{\sigma_{\text {inel }}}
$$

where $\mu$ is the average number of inelastic interactions per bunch crossing (called pile-up parameter). Thus, the instantaneous luminosity can be determined using any method that measures the ratio $\mu / \sigma_{\text {inel }}$.

Techniques for luminosity determination can be classified as follows:

- Event Counting: the fraction of bunch crossings during which a detector registers an event satisfying a given selection requirement is counted. A bunch crossing can be said to contain an event if at least one pp interaction in that crossing induces at least one observed hit in the detector being considered.

- Hit Counting: the number of hits (for example, electronic channels or clusters with energy above a specified threshold) per bunch crossing in a given detector is counted.

- Particle Counting: luminosity is proportional to the distribution of the number of particles per beam crossing (or its mean value). That number can be inferred from reconstructed quantities (e.g. tracks), from pulse-height distributions, or from other observables that reflect the instantaneous particle flux traversing the detector. 
Equation 2.2 can be rewritten as:

$$
L=\frac{\mu n_{b} f_{r}}{\sigma_{\text {inel }}}=\frac{\mu_{v i s} n_{b} f_{r}}{\varepsilon \sigma_{\text {inel }}}=\frac{\mu_{v i s} n_{b} f_{r}}{\sigma_{v i s}}
$$

where $\varepsilon$ is the efficiency for one inelastic pp collision to satisfy the event-selection criteria, and $\mu_{v i s}=\varepsilon \mu$ is the average number of visible inelastic interactions per bunch crossing (i.e. the mean number of pp collisions per bunch crossing that pass that event selection). The visible cross section $\sigma_{v i s}=\varepsilon \sigma$ is the calibration constant that relates the measurable quantity to the absolute luminosity.

\section{Calibration during the van der Meer scans}

The most common technique to measure $\sigma_{v i s}$ is to evaluate the absolute luminosity inferred from measured accelerator parameters:

$$
L=\frac{n_{b} f_{r} n_{1} n_{2}}{2 \pi \Sigma_{x} \Sigma_{y}}
$$

where $n_{1}$ and $n_{2}$ are the numbers of particles in the two colliding bunches, and $\Sigma_{x}$ and $\Sigma_{y}$ characterize the widths of the horizontal and vertical beam profiles. $\Sigma_{x}$ and $\Sigma_{y}$ are typically measured during van der Meer (vdM) scans (also called beam-separation or luminosity scans) [3]. The observed event rate is recorded while scanning the two beams across each other in the two directions. This measurement yields two bell-shaped curves, with the maximum rate at zero separation, from which the values of $\Sigma_{x}$ and $\Sigma_{y}$ are extracted. The luminosity at zero separation can then be computed using Equation 3.1, and $\sigma_{v i s}$ extracted from Equation 2.3 using the values of $L$ and $\mu_{v i s}$ measured by each detector.

\section{ATLAS Luminosity Monitors}

The ATLAS detector is discussed in detail in [1]. The two primary luminometers, BCM (Beam Conditions Monitor) and LUCID (LUminosity measurement using a Cherenkov Integrating Detector), both perform deadtime-free, bunch-by-bunch luminosity measurements. These are compared with the results of the track-counting method, a new approach developed by ATLAS which monitors the multiplicity of charged particles produced in randomly selected colliding-bunch crossings. Additional methods have been developed to disentangle the relative long-term drifts and run-torun variations between LUCID and track-counting measurements during high-luminosity running, thereby reducing the associated systematic uncertainties to the sub-percent level. These techniques measure the total instantaneous luminosity, summed over all bunches. The first technique measures the particle flux from pp collisions as reflected in the current drawn by the PMTs of the hadronic calorimeter (TileCal). This flux, which is proportional to the instantaneous luminosity, is also monitored by the total ionization current flowing through a well-chosen set of liquid-argon (LAr) calorimeter cells. A third technique, using Medipix radiation monitors (TPX), measures the average particle flux observed in these devices. During the full Run 2, from 2015 on, LUCID was the ATLAS preferred online luminosity monitor. More details can be found in [4]. 


\section{CMS Luminosity Monitors}

A total of five systems are used for measuring luminosity at CMS [2]. Three of them are used for online measurements: the Pixel Luminosity Telescope (PLT) and the Fast Beam Conditions Monitor (BCM1F) exploit an event/hit counting algorithm. The third detector, the Hadronic Forward calorimeter (HF), uses two different approaches: the HFOC algorithm is a hit counting method, while the HFET algorithm extrapolates the luminosity from energy flow measurements. Two other methods, the Drift Tube luminosity (DT) and Pixel Cluster Counting (PCC), are used for offline measurements and cross checks. More details on luminosity monitors and methods can be found in [5]. For 2017 data, the luminosity measurement is based on HFET algorithm, complemented with PCC.

\section{Systematic Uncertainties}

\begin{tabular}{|c|c|c|}
\hline vdM calibration uncertainties & ATLAS & CMS \\
\hline \multirow[t]{5}{*}{ Bunch-charge product } & Beam current calibration & Beam current calibration \\
\hline & Ghost and satellites & Ghost and satellites \\
\hline & Orbit-drift correction & Orbit-drift correction \\
\hline & Beam position jitter & - \\
\hline & Emittance growth correction & - \\
\hline \multirow[t]{7}{*}{ Beam conditions } & Scan-to-scan reproducibility & Scan-to-scan reproducibility \\
\hline & Bunch-to-bunch consistency & Bunch-to-bunch consistency \\
\hline & Fit model & - \\
\hline & Non-factorization effects & Non-factorization effects \\
\hline & Beam-beam effects & Beam-beam effects \\
\hline & Cross-detector consistency & Cross-detector consistency \\
\hline & Background subtraction & - \\
\hline \multirow[t]{2}{*}{ Instrumental effects } & Length scale calibration & Length scale calibration \\
\hline & ID length scale & - \\
\hline
\end{tabular}

Table 1: Full list of vdM calibration related uncertainties, considered by ATLAS or CMS.

\begin{tabular}{|c|c|c|}
\hline L monitoring uncertainties & ATLAS & CMS \\
\hline Monitoring & Internal stability & Internal stability \\
& Linearity & Linearity \\
& Afterglow & Afterglow \\
& - & Afterpulses \\
& - & Dead time \\
\hline
\end{tabular}

Table 2: Full list of luminosity monitoring related uncertainties, considered by ATLAS or CMS.

Some of the main systematic uncertainties affecting the final luminosity value are briefly described below. Full lists of uncertainties are given in tables 1 (calibration related) and 2 (luminosity 
monitor related): they will not be described here but still make variuos contributions to the total uncertainty.

- Linearity. The response of the luminometer could shift between vdM scans, performed at very low luminosity, low $\mu$ values and few widely-separated bunches, and physics runs, typically performed at high luminosity and $\mu$ values with more than 2000 bunches organized in trains of bunches $25 \mathrm{~ns}$ apart. For ATLAS in 2017 a typical non-linearity correction, obtained by comparing LUCID and tracks results, is about $9 \%$ at $\mu=50$ with an associated uncertainty of $\pm 1.3 \%$ (see figure 1). For CMS in 2017 both correction and systematic uncertainties are equal to $1.5 \%$.

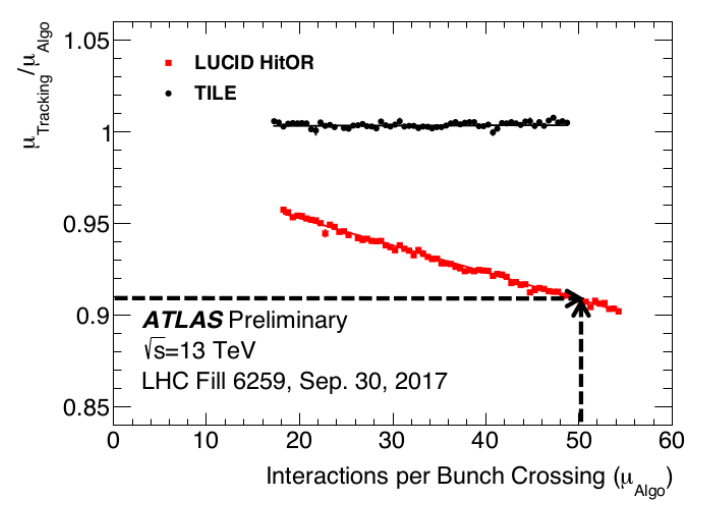

Figure 1: Ratio of LUCID HitOR algorithm (red) and Tile (black) to Tracks (not affected by non-linearity). The correction for non-linearity effects can be inferred fitting LUCID data [6].

- Consistency of bunch-by-bunch visible cross-sections. The calibrated $\sigma_{v i s}$ value found for a given detector and algorithm should be a constant factor independent of machine conditions or choice of different BCIDs. The comparison between the $\sigma_{v i s}$ values determined by BCID demonstrated that there is some degree of scatter between the values not entirely of statistical origin. The amount of the uncertainty in 2017 is $\pm 0.9 \%$ for CMS and $\pm 1.2 \%$ for ATLAS (see figure 2).

- Non-factorization effects. The vdM formalism outlined in Sect. 3 explicitly assumes that the particle densities in each bunch can be factorized into independent horizontal and vertical components such that the term $1 /\left(2 \pi \Sigma_{x} \Sigma_{y}\right)$ in equation 3.1 fully describes the overlap integral of the two beams. If the factorization assumption is violated, the convolved beam width $\Sigma$ in one plane is no longer independent of the beam separation $\delta$ in the other plane, although a straightforward generalization of the vdM formalism still correctly handles an arbitrary two-dimensional luminosity distribution as a function of the transverse beam separation $\left(\delta_{x} \delta_{y}\right)$, provided this distribution is known with sufficient accuracy. Linear x-y correlations do not invalidate the factorization assumption, but they can rotate the ellipse which describes the luminosity distribution away from the $x-y$ scanning planes such that the measured $\Sigma_{x}$ and $\Sigma_{y}$ values no longer accurately reflect the true convolved beam widths. The corresponding correction and uncertainty in 2017 are $0.8 \pm 0.8 \%$ for ATLAS and $0.2 \pm 0.2 \%$ for CMS. 


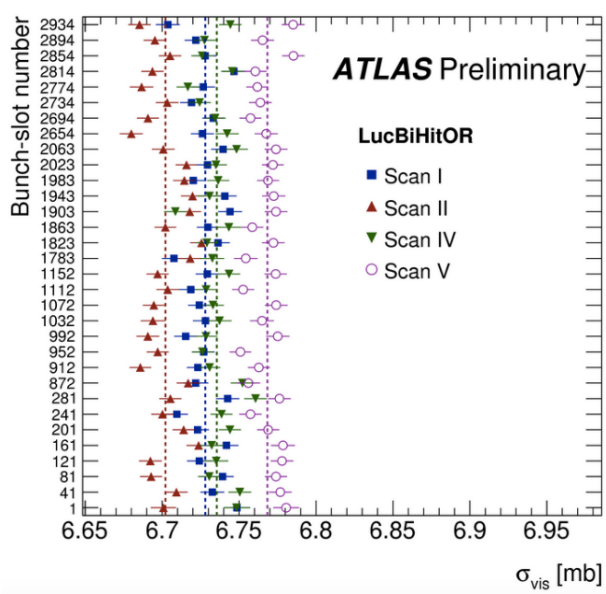

Figure 2: Scan-to-scan reproducibility of vdM calibration for ATLAS in 2017 [6].

- Long term stability. A fundamental ingredient of the experiment strategy to assess and control the systematic uncertainties affecting the absolute luminosity determination is to compare the measurements of several luminosity detectors and algorithms, that are characterized by different acceptance, response to pile-up, and sensitivity to instrumental effects and to beam-induced backgrounds. The level of consistency across the various methods, over the full range of single-bunch luminosities and beam conditions, provides valuable cross-checks as well as an estimate of the detector-related systematic uncertainties. For the 2017 data taking, the stability uncertainty is $\pm 1.3 \%$ for ATLAS, obtained from the envelope of all available relative-luminosity monitors over the entire period. The stability uncertainty for CMS in 2017 is $\pm 0.5 \%$, evaluated from the RMS of the ratio of the two monitors providing 99.4\% of the total 2017 luminosity (HFET and PCC) (see figure 3). During 2017 CMS developed a powerful tool to assess and control linearity during runs, called emittance scan (see next section).
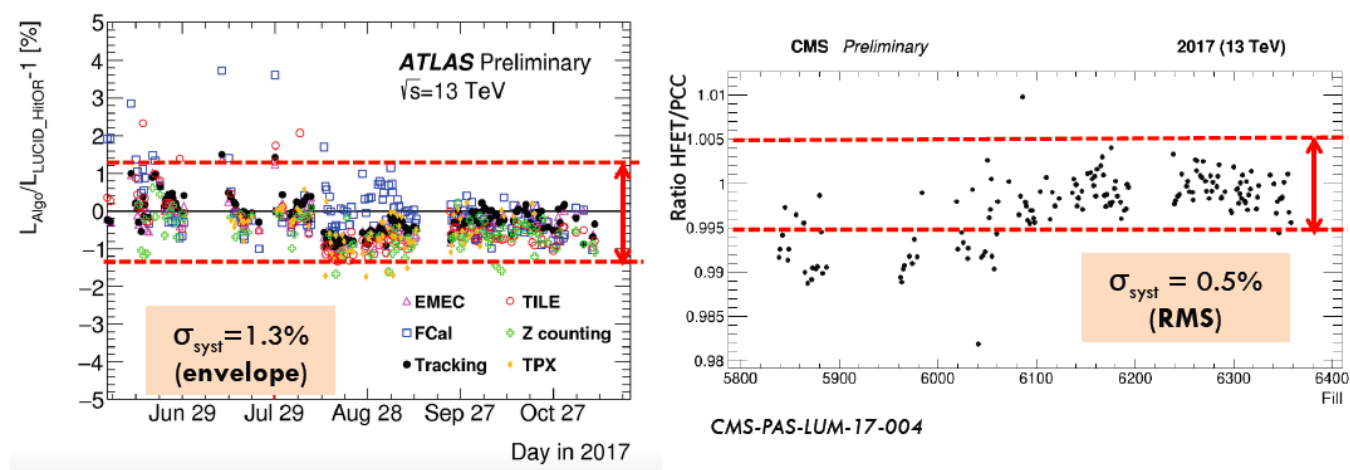

Figure 3: Long term stability for ATLAS (left) [6], obtained comparing all available relative-luminosity monitors over the entire period, and for CMS (right), obtained from the RMS of the ratio of HFET and PCC [5]. 


\section{Emittance scans}

The emittance scans are short vdM-like scans performed at the beginning and at the end of LHC fills in standard physics conditions (see figure 4). Although emittance scans have a lower level of precision than vdM scan due to a more limited scanning range, possible non factorization biases, and beam dynamics effects, they are still useful to assess linearity and stability effects as well as to cross check the performace of the luminosity monitors during runs. The success of CMS emittance scans motivates ATLAS to perform the same scans during 2018.

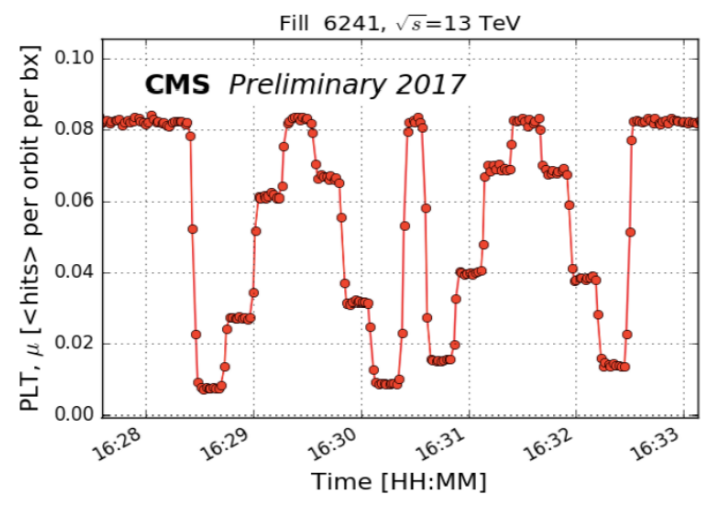

Figure 4: Instantaneous luminosity measured during the emittance scan performed in fill 6241 at $13 \mathrm{TeV}$ of center-of-mass energy in 2017. Beams are scanned in X and Y planes in 7/9 displacement steps of 10s/point [7].

\section{Conclusions}

Precision measurement of the luminosity delivered to the experiments ATLAS and CMS by the LHC is important for both online and offline purposes. The preliminary total luminosity uncertainty obtained in 2017 is $2.4 \%$ for ATLAS and 2.3\% for CMS, which is an impressive achievement for a hadron collider but still dominant for some cross section measurements.

\section{References}

[1] ATLAS Collaboration, The ATLAS Experiment at the CERN Large Hadron Collider, JINST 3 (2008) S08003

[2] CMS Collaboration, The CMS Experiment at the LHC, JINST 3 (2008) S08004

[3] S. van der Meer, Calibration of the Effective Beam Height in the ISR, CERN-ISR-PO-68-31

[4] ATLAS Collaboration, Luminosity determination in pp collisions at $\sqrt{s}=8 \mathrm{TeV}$ using the ATLAS detector at the LHC, Eur. Phys. J. C 76 (2016) 653

[5] CMS Collaboration, https://cds.cern.ch/record/2621960

[6] ATLAS Collaboration, http://atlas.web.cern.ch/Atlas/GROUPS/PHYSICS/PLOTS/LUMI-2017-001

[7] CMS Collaboration, https://cds.cern.ch/record/2306378 